\title{
Irritativ-toxische Dermatitis und Exazerbation einer chronischen Plaque-Psoriasis unter Anwendung von Regividerm ${ }^{\circledR}$
}

\author{
Toxic Dermatitis and Worsening of a Chronic Plaque Psoriasis After Treatment with a Vitamin B(12) Ointment \\ Containing Avocado Oil
}

Autoren

Institut
T. Glaenz, L. Bröhl, U. Amon

PsoriSol-Klinik für Dermatologie \& Allergologie, Hersbruck

\section{Bibliografie}

DOI http://dx.doi.org/

10.1055/s-0029-1244182

Akt Dermatol 2010; 36:

278-280 @ Georg Thieme

Verlag KG Stuttgart · New York

ISSN 0340-2541

Korrespondenzadresse Dr. med. Thomas Glaenz Oberarzt der PsoriSol-Hautklinik Mühlstr. 31

91217 Hersbruck

glaenz@psorisol.de

\section{Zusammenfassung \\ $\nabla$}

In einer Fernsehsendung der ARD wurde Regividerm $^{\circledast}$ als praktisch nebenwirkungsfreie Option zur Behandlung von sowohl Psoriasis als auch atopischem Ekzem gepriesen. Ein 43-jähriger Patient mit chronischer Plaque-Psoriasis hatte über 4-5 Tage Regividerm-Creme zur Lokaltherapie angewendet und hierunter eine rasche Verschlechterung des Befundes bemerkt. Bei Aufnahme zeigte sich das Bild einer erosiven und exsudativen Kontaktdermatitis an den Streckseiten der Extremitäten. Das Routinelabor war unauffällig; die Histologie ergab eine irritierte Psoriasis mit Spongiose und Exoserose. Zunächst rasche Stabilisierung unter antibiotischer und externer adstringierender und desinfizierender Therapie; dann Anwendung von Vitamin $\mathrm{D}_{3}$-Analoga und Fototherapie, zusätzlich Methotrexat-Kurzzeittherapie. Entgegen der öffentlichkeitswirksam gepriesenen Nebenwirkungsfreiheit kann eine erhebliche toxisch-irritative Dermatitis mit nachfolgender Köbnerung der Psoriasis auftreten.

\section{Einleitung}

In einer im Oktober 2009 im abendlichen ARDFernsehprogramm ausgestrahlten Reportage wurde eine Vitamin $B_{12}$-haltige Salbe (Regivi$\operatorname{derm}^{\circledR}$ ) als wirksam und insbesondere praktisch nebenwirkungsfrei für die Therapie von atopischem Ekzem oder Psoriasis gepriesen.

Wir berichten über einen Patienten mit chronisch-stationärer Plaque-Psoriasis, der nach mehrtägiger Anwendung von Regividerm ${ }^{\circledR}$ eine Exazerbation des Befundes und eine starke toxisch-irritative Dermatitis an den Applikationsorten erlitten hat, welche ihrerseits eine Ausbreitung der Psoriasis in den Arealen der abheilenden Kontaktdermatitis (Köbner-Phänomen) nach sich zog und eine stationäre systemische Behandlung erforderlich machte.

\section{Anamnese}

Bei dem 43-jährigen, sonst gesunden Patienten besteht seit 18 Jahren eine chronisch-stationäre Psoriasis vulgaris der Prädilektionsstellen. Der Befund war ambulant unter topischer Therapie bisher gut kontrolliert; Arthralgien bestehen nicht. Ende November 2009 wurde von dem Pa- tienten selbst Regividerm ${ }^{\circledR}$-Salbe erprobt: Innerhalb von 4-5 Tagen kam es zu einer disseminierten Aussaat frischer psoriatischer Plaques am Stamm, zusätzlich zu einer raschen Größenprogredienz der behandelten münzgroßen PsoriasisPlaques an den Unterarmen und unterhalb des rechten Knies, nachfolgend dort zu einer starken düsteren, großflächigen Rötung mit Schmerzhaftigkeit der Haut. Der rechte Unterschenkel schwoll an. Regividerm ${ }^{\circledR}$-Creme wurde abgesetzt und der Hausarzt konsultiert, der den Patienten unverzüglich in unsere Klinik einwies.

\section{Aufnahmebefund \\ $\nabla$}

An den Streckseiten beider Unterarme ( $\bullet$ Abb. 1) und am rechten Unterschenkel ( $\bullet$ Abb. 2) ventral großflächiges Erythem mit glänzend prall gespannter Haut, feinen Rhagaden, Exsudation und randbetonter groblamellärer Abschilferung. Der rechte Unterschenkel weist gegenüber dem linken eine ödematöse Schwellung mit Umfangsvermehrung auf. Daneben an der behaarten Kopfhaut und am Körperstamm zahlreiche tropfenbis münzgroße typische erythematosquamöse Plaques. 


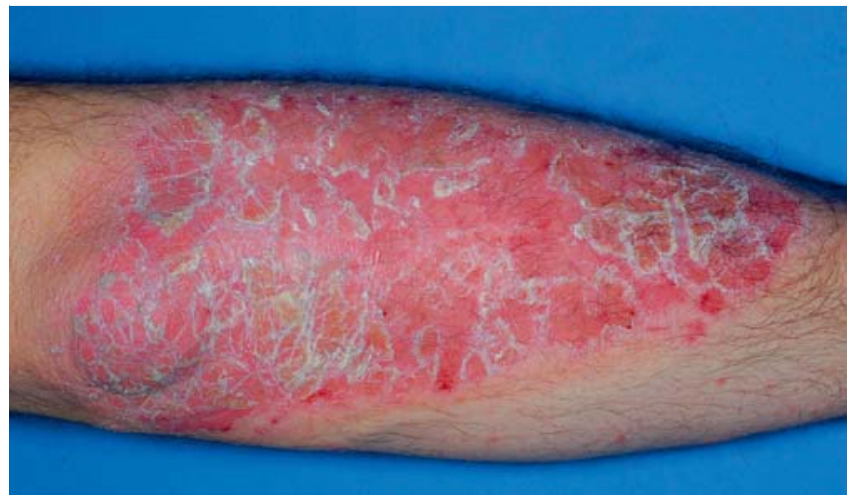

Abb. 1 Aufnahmebefund am rechten Unterarm.

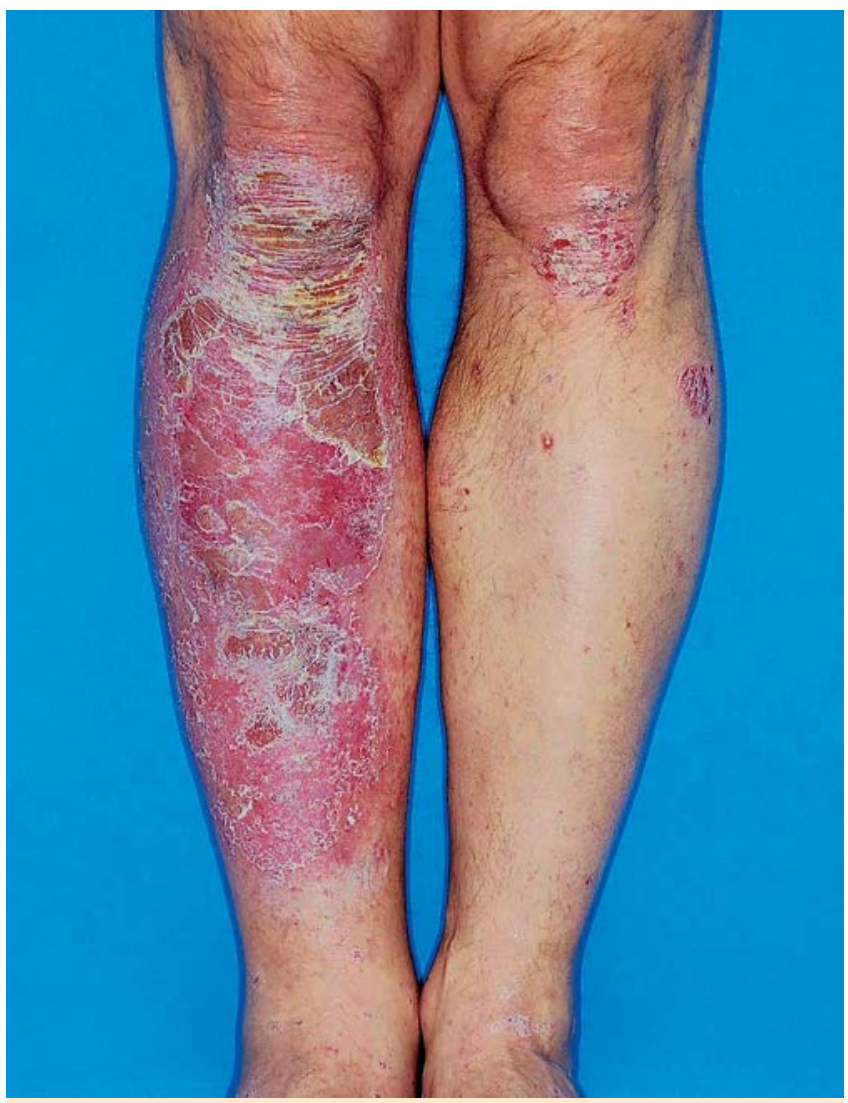

Abb.2 Aufnahmebefund am rechten Unterschenkel.

\section{Befunde diagnostischer Untersuchungen}

$\nabla$

Orientierendes Labor einschließlich serologischer Entzündungsparameter unauffällig. In der Histologie vom rechten Unterarm zeigte sich ein mit einer irritierten Psoriasis vulgaris vereinbarer Befund mit Nachweis von Exoserose und Spongiose.

\section{Therapie und Verlauf}

$\nabla$

Die frischen psoriatischen Plaques behandelten wir nach 2-tägiger Keratolyse mit Calcitriol-Salbe und führten eine GanzkörperBade-PUVA durch.

Die toxische Dermatitis an den Extremitäten wurde über mehrere Tage mit Kaliumpermanganat-Umschlägen und einer Betame-

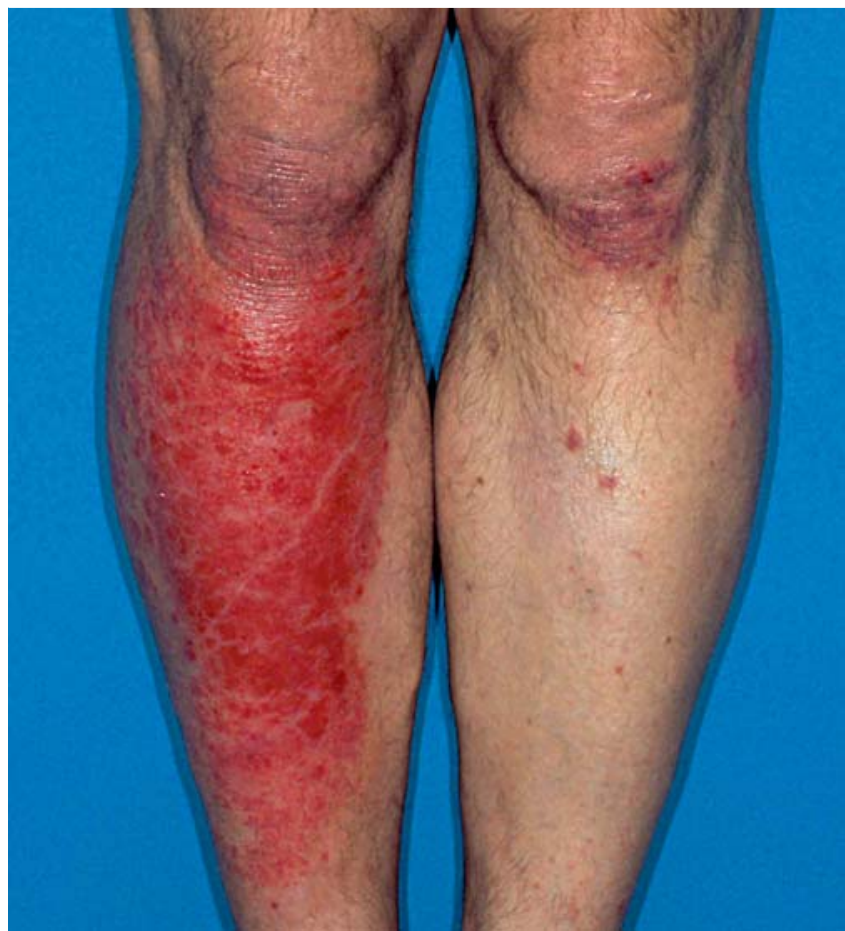

Abb. 3 Frische psoriatische Infiltrate im Areal der vorangegangenen Kontaktdermatitis.

thason-Lotio behandelt, der rechte Unterschenkel mit Kurzzugbinden tagsüber kompressionstherapiert. Nach dem Rückgang des Erythems dann Fortführung der topischen Therapie mit Hydrocorticonaceponat bis zur klinischen Abheilung. Initial zudem wegen klinischer Zeichen der Superinfektion antibiotische Abdeckung mit Cefaclor über 5 Tage.

Unter dieser Therapie kam es zu einer raschen Besserung der Kontaktdermatitis; allerdings traten infolge des starken Entzündungsreizes im Sinne eines Köbner-Phänomens neue, flächenhafte psoriatische Plaques ( $\mathbf{A b b} \mathbf{3}$ ) in den Arealen der abklingenden Kontaktdermatitis auf, weshalb zuletzt noch eine Kurzzeittherapie mit Methotrexat eingeleitet wurde.

\section{Diskussion}

\section{$\nabla$}

Das als Regividerm ${ }^{\circledR}$-Salbe zugelassene Medizinprodukt enthält $0,07 \%$ Vitamin $B_{12}$ und $46 \%$ Avocadoöl.

Die Wirksamkeit des Vitamins $B_{12}$ soll auf der Bindungsfähigkeit von Stickstoffmonoxid beruhen $[3,5]$, welchem eine wichtige Rolle in der Pathogenese zahlreicher entzündlicher Dermatosen zugeschrieben wird [2]. Als Beleg dienen 3 Publikationen [3-5] hierzu. Bei der ältesten aus dem Jahre 2001 wurde die Wirksamkeit mittels Seitenvergleich mit Calcipotriol an 13 Patienten mit chronischer Plaque-Psoriasis über 12 Wochen untersucht. Hierbei sei Calcipotriol in den ersten 4 Wochen des Beobachtungszeitraumes überlegen gewesen, der Effekt habe dann jedoch nachgelassen; die Vitamin $\mathrm{B}_{12}$-Zubereitung habe jedoch einen über 12 Wochen konstanten Effekt gezeigt und sei signifikant besser vertragen worden [4]. Angesichts der Prävalenz der Plaque-Psoriasis in der Bevölkerung stellt sich hier die Frage, weshalb nur ein so kleines Patientenkollektiv ausgewählt wurde, anhand dessen sich kaum gültige Aussagen zu Wirksamkeit und Verträglichkeit einer Therapie machen lassen. 
Der zweiten Publikation aus dem Jahre 2004 liegt eine prospektive, randomisierte, plazebokontrollierte, multizentrische Studie anhand von 49 Patienten mit atopischer Dermatitis zugrunde. Auch hier erfolgte ein Seitenvergleich, der Therapieerfolg wurde anhand des SASSAD-(Six area six sign atopic dermatitis severity-) Score gemessen und die Einschätzung von Patient und Studienarzt einbezogen, welche zu der Folgerung führte, dass topisches Vitamin $B_{12}$ eine gegenüber Plazebo wirksamere und sehr gut tolerierte, für den Patienten mit sehr niedrigem Risiko behaftete Therapieoption sei [3]. Allerdings wurden in dieser Studie bei immerhin 33 Patienten Hautreaktionen verzeichnet, welche bei 2 von ihnen zum Therapieabbruch führten. Darüber hinaus ist der SASSAD-Score unzureichend validiert und wie viele andere Scores deutlichen interindividuellen Schwankungen unterworfen [6].

Zuletzt wurde 2009 eine Untersuchung an Kindern mit atopischem Ekzem (doppelblinde, randomisierte, plazebokontrollierte Studie) im Journal of Alternative and Complementary Medicine publiziert, bei denen über lediglich 4 Wochen der Therapieerfolg und die Verträglichkeit dokumentiert wurde. Auch hier sah man eine signifikante Besserung gegenüber Plazebo [5]. Das Organ versteht sich expressis verbis als Plattform abseits der Wege der evidenzbasierten Schulmedizin.

Die Zulassung erfolgte im Oktober 2009 als Medizinprodukt und nicht unter den strengeren behördlichen Kautelen als Arzneimittel.

Versuche mit Vitamin $\mathrm{B}_{12}$-haltigen Externa sind nicht neu; vor einigen Jahren haben wir bei hiermit einverstandenen Patienten nach umfassender Aufklärung in unserer Klinik selbst eine Vitamin $B_{12}$-haltige Creme angewendet, wobei zwar keine nennenswerten unerwünschten Effekte auftraten, insgesamt aber die Wirkung enttäuschend war und die einer hochwertigen indifferenten Hautpflege nicht übertraf.

Aus unserer Sicht deutet die wissenschaftlich dürftige Studienlage für Regividerm ${ }^{\circledR}$ eher auf Wirkungslosigkeit als auf eine im Fernsehen publikumswirksam vermarktete „Wundersalbe“ hin [1].

Besonders marktwirksam war sicherlich das Argument der Nebenwirkungsfreiheit, zumal Patienten mit Psoriasis oder atopischem Ekzem nach langjähriger Anwendung topischer Steroide mit den hinlänglich bekannten Nebenwirkungen oder nach dem Erleben von Hautirritationen und Missempfindungen nach Anwendung topischer Calcineurininhibitoren oder Vitamin $\mathrm{D}_{3}$-Analoga hierfür sehr empfänglich sind.

Dass schon in einer der drei Studien [3] immerhin bei 33 von 49 Patienten mit atopischem Ekzem Hautirritationen aufgetreten waren und 2 von diesen Patienten deshalb die Behandlung ganz abgebrochen haben, wurde wohl mit Rücksicht auf die gewünschte Sensation des Fernsehberichts kaum thematisiert. Immerhin werden „,vorübergehende“ Hautrötungen, die nicht zu einem Therapieabbruch führen müssen, im Beipackzettel erwähnt.

Unser Fall zeigt, dass keinesfalls von einer „Nebenwirkungsfreiheit" gesprochen werden kann. Selbst wenn konzediert wird, dass der zeitliche Zusammenhang der Exazerbation der Psoriasis bei unserem Patienten mit der Applikation von Regividerm ${ }^{\circledR}$ nicht zwingend den Schluss auf einen kausalen Zusammenhang zulässt, so bleibt doch das Faktum einer hochakuten irritativ-toxischen Dermatitis an den Applikationsorten, welche letztlich zur stationären Aufnahme führte und im weiteren Verlauf eine (Erst-)Manifestation der Psoriasis im Bereich der abklingenden Kontaktdermatitis i.S. eines Köbner-Phänomens bedingte.

Anhand des präsentierten Falles wird deutlich, dass eine toxische Kontaktreaktion auftreten und nicht unerhebliche Folgen (stationärer Aufenthalt, Antibiose und Systemtherapie der Psoriasis) für den Patienten selbst, aber auch für das Gesundheitssystem nach sich ziehen kann.

Zusammenfassend kann bis zu einer möglichen Zulassung von Regividerm $^{\circledR}$-Salbe als Arzneimittel, versehen mit entsprechenden Warnhinweisen auf mögliche unerwünschte Effekte, insbesondere aber auch bis zum Vorliegen weiterer valider Studien anhand größerer Patientenkollektive von der Anwendung dieses Medizinprodukts aus unserer Sicht nur abgeraten werden.

\section{Abstract}

\section{Toxic Dermatitis and Worsening of a Chronic Plaque Psoriasis After Treatment with a Vitamin B(12) Oint- ment Containing Avocado Oil \\ $\nabla$}

Vitamin $\mathrm{B}(12)$ ointment containing avocado oil was praised as nearly free from side effects for the treatment of psoriasis or atopic dermatitis in the German telecast.

We report on a 43-years-old patient with chronic plaque psoriasis who used the ointment for about five days. Rapid worsening of the treated areas occurred.

At the day of admission we saw an erosive weeping dermatitis on the limbs.

Routine blood samples were negative, histological findings revealed an irritated psoriasis with spongiosis and exoserosis. Under an initial systemic antibiotic therapy, local disinfection and topical corticosteroid treatment followed by the use of calcitriol, phototherapy and a short time oral therapy with methotrexate it came to a rapid improvement. Our case shows that in contrast to the report in the telecast Regividerm ${ }^{\circledR}$ has a risk for the development of considerably strong side effects such as local toxic contact dermatitis.

\section{Literatur}

1 Vorsicht Desinformation. Regividerm: ARD als Marketinghelfer. Arznei-Telegramm 2009; 40: 99

2 Bruch-Gerharz D, Ruzicka T, Kolb-Bachofen V. Nitric oxid in human skin: current status and future prospects. J Invest Dermatol 1998; 110: $1-7$

3 Stücker M, Pieck C, Stoerb C et al. Topical vitamin B12 - a new therapeutic approach in atopic dermatitis - evaluation of efficacy and tolerability in a randomised placebo-controlled multicentre clinical trial. $\mathrm{Br} \mathrm{J}$ Dermatol 2004; 150: 977 - 983

4 Stücker M, Memmel U, Hoffmann $M$ et al. Vitamin B(12) cream containing avocado oil in the therapy of plaque psoriasis. Dermatology 2001; 203: $141-147$

5 Januchowski R. Evaluation of topical vitamin $\mathrm{B}(12)$ for the treatment of childhood eczema. J Altern Complement Med 2009; 15: 387-389

6 Charman CR, Venn AJ, Williams HC. Reliability testing of the Six Area, Six Sign Atopic Dermatitis severity score. Br J Dermatol 2002; 146: $1057-1060$ 